\title{
A finitude humana e a saúde pública
}

\author{
Human finitude and public health
}

\author{
1 Escola Nacional de Saúde \\ Pública Sergio Arouca, \\ Fundação Oswaldo Cruz, \\ Rio de Janeiro, Brasil. \\ 2 Faculdade de Medicina, \\ Universidade Federal \\ do Rio de Janeiro, \\ Rio de Janeiro, Brasil. \\ Correspondência \\ S. Rego \\ Departamento de Ciências \\ Sociais, Escola Nacional de \\ Saúde Pública Sergio Arouca \\ Fundação Oswaldo Cruz. \\ Rua Leopoldo Bulhões 1480, \\ Rio de Janeiro, $R J$ \\ 21041-210, Brasil. \\ rego@ensp.fiocruz.br
}

\section{Abstract}

Death is an implicit subject in both health practice and health professionals' training. There is a tendency by health professionals to reduce this analysis merely to technical aspects. This paper analyzes the importance of adopting quality care at the end of life as a guide for public health care. We highlight relevant aspects for training health professionals to respect moral differences and to be prepared to discuss their own points of view.

Hospice Care; Health Policy; Quality of Life; Bioethics
Sergio Rego 1

Marisa Palácios 2

\section{Introdução}

A morte é um tema presente, explicitamente ou não, nas discussões sobre a assistência e promoção da saúde e organização de serviços. Mas é também um assunto a não ser nominado, seja no âmbito familiar, seja no profissional. A morte ainda é vista, por muitos profissionais de saúde, como um fracasso, incapacidade ou incompetência, uma vez que eles foram formados para combatê-la. A incerteza, parceira constante no exercício profissional, é uma coadjuvante da insegurança e um estímulo para a resistência ao "êxito letal". Mas, como diz o dito popular, a morte é a única coisa certa na vida, de tal forma que se pode considerar que é a consciência da própria morte o que nos humaniza. O morrer deixou de ser um fenômeno doméstico e passou a ser público ${ }^{1}$, passando a morte do ambiente familiar ao hospitalar. Se os indivíduos passaram a morrer no hospital, pois sua medicalização levou os enfermos para lá na esperança de cura, nos dias atuais, a dependência tecnológica e a pressão dos custos de internação tensionam essa decisão. Não obstante haver claramente um movimento internacional de (re)valorização da morte na residência do enfermo, é razoável levantar-se dúvidas sobre qual dos benefícios de tal posição seja seu principal fundamento: se a desejada "humanização" do processo de morrer 2 ou se a redução de custos a ele relacionados. 
O desenvolvimento biotecnológico e a revolução cultural ética 3 tornaram obsoletas as tradicionais respostas baseadas no princípio da sacralidade da vida. Assim, outros princípios passaram a ser levados em consideração na tomada de decisões, assumindo especial importância a discussão sobre a qualidade da vida. Entendemos com Schramm 4 que, considerando seriamente o pluralismo moral e ético vigente nas sociedades complexas, não cabe fazer uma tentativa de síntese entre os princípios da sacralidade da vida e da qualidade da vida. "Repensar a vida em termos de suas 'qualidades' e 'nudez' ou 'desamparo' constitui uma condição para começar a pensar também, em âmbito crítico, os desafios que a biotecnociência parece colocar para a Bioética" 4 (p. 21), entendida como uma ferramenta que pode defender tanto “'a vida nua' quanto a vida considerada do ponto de vista da 'sacralidade' e/ou da 'qualidade”" 4 (p. 21). Entendendo que a proteção do bem-estar de populações é uma das preocupações essenciais do campo da Saúde Coletiva, a Bioética inscreve, no campo, também a preocupação com o bem-estar do indivíduo, lembrando que não se deve perder de vista a individualidade quando se observa a coletividade e nem a coletividade quando se observa o indivíduo 5 .

Trata-se aqui de propor e entender o cuidado como norteador da assistência aos indivíduos no final de suas vidas. Para tal, discutiremos o próprio conceito de cuidado no âmbito da assistência à saúde para, em seguida, buscar delinear de que forma a questão do cuidado no final da vida é e precisa ser tomada como objeto da Saúde Coletiva, além das profissões diretamente envolvidas com a prestação dos serviços. Procuraremos identificar de que forma os profissionais da Saúde Coletiva têm considerado e se posicionado em relação às questões relacionadas ao fim da vida para defendermos medidas estratégicas e urgentes na preparação de profissionais e técnicos de saúde aptos a discutirem o cuidado não só em uma perspectiva de assistência multidisciplinar, mas também com o compromisso do diálogo como eixo condutor na tomada de decisões.

\section{Fim da vida e assistência à saúde}

É preciso encarar não a morte como evento, mas o processo de morrer, como afirmaram, entre outros, Siqueira-Batista \& Schramm 6 (p. 38): “não é a morte o que realmente importa, mas sim o seu processo, a certeza de que a vida se enveredou por um 'caminho' sem volta". As- sim, não é relevante também definir objetivamente o início desse "caminho".

O cuidado emerge como uma questão na qualidade do fim da vida nos anos 90 7. Cuidados no fim da vida englobam um conjunto de teorias e práticas que têm por objeto central o processo de morrer. Não deve ser uma prática restrita a especialistas, mas um dos marcos principais no processo de formação da equipe de saúde. A qualidade do fim da vida demanda cuidados específicos de diversos profissionais, que lidam com o "caminho sem volta" e que é impossível precisar a duração ou mesmo o momento de início. Doenças graves/crônicas que levam à morte também acometem crianças $\mathrm{e}$ adolescentes. O processo de morrer também deve ser objeto de cuidado nesse caso. E que tipo de cuidado é esse? Em uma pesquisa 7, especialistas em cuidados paliativos identificaram seis elementos considerados chaves: qualidade de vida geral; desempenho e bem-estar físico; desempenho e bem-estar psicossocial; bem-estar espiritual; a percepção do paciente acerca do cuidado; as percepções e o bem-estar da família. Pesquisas posteriores mostraram que há diferenças significativas quando a pergunta é dirigida a especialistas em cuidados paliativos, a outros profissionais de saúde ou pacientes 7. É que a perspectiva do cuidado deve ser uma perspectiva dialógica. O cuidado é algo a ser compartilhado. Pressupõe o respeito à autonomia dos sujeitos na perspectiva da proteção e em seu contexto social e familiar: considerando o estado de destituição no qual uma considerável parte da população se encontra, há que haver, por parte dos profissionais e do sistema de saúde, ações que possam minimizar ou corrigir algumas das circunstâncias que tornam essas pessoas especialmente vulneráveis.

Cabe ao campo da Saúde Coletiva/Saúde Pública contribuir para o planejamento de serviços e sistemas de saúde que contemplem a questão do cuidado no fim da vida, assim como formular e contribuir na implementação de políticas setoriais específicas, inclusive na formação de recursos humanos em saúde. Mas essa discussão não pode dar-se em um vazio moral, fundamentado apenas na hegemonia técnica. Nela, não se incluem, de fato, as diferentes questões morais e nem o conjunto de temas que são mantidos camuflados no cotidiano da assistência, deixados, muitas vezes, apenas ao arbítrio de um ou outro profissional de saúde.

Císio Brandão destacou que está cada vez mais clara a idéia que ele chamou de "cuidado total aos pacientes", assinalando que "cuidados paliativos não são apenas uma opção terapêu- 
tica, mas sim um direito de todos que objetivam uma melhor qualidade de vida diante de situações potencialmente ameaçadoras (...), não apenas no final da vida, mas em todas as fases da vida e no transcurso de doenças ainda ditas como incuráveis, como a grande maioria dos cânceres, HIVIAIDS, DPOC, insuficiência cardíaca, doenças neurológicas degenerativas, em geriatria e outras, onde o foco principal do tratamento é a qualidade de vida" 8. A maioria das unidades hospitalares brasileiras não apenas não possui uma diretriz sobre como cuidar de seus pacientes que estejam com um quadro terminal, mas também não há informações sistematizadas sobre como esses últimos momentos são vividos, seja pelos pacientes, seja por seus familiares, com as exceções de praxe 9 .

Destaca-se também a Política Nacional de Humanização que, nos últimos três anos, orienta esforços pela valorização dos sujeitos envolvidos no processo de produção de saúde e fomenta a autonomia e o protagonismo desses sujeitos 10 . As ações de humanização promovidas pelo Ministério da Saúde têm ampliado as discussões em torno do respeito ao usuário e aos trabalhadores dos serviços e, em alguns hospitais, têm atuado fomentando atenção integral ao fim de vida. Mas ainda estamos muito longe de atingirmos tais propósitos. A integralidade preconizada é vista como imagem-objetivo 11 multifacetada, significando a integração das ações curativas, reabilitadoras, preventivas, em benefício da qualidade de vida do sujeito em sua rede de apoio.

\section{Racionalidade como ilusão}

A formação em saúde prepara o indivíduo para lidar com três tipos de incertezas 12: a incerteza sobre se o que o profissional não sabe é decorrente do pouco estudo e pequena experiência ou se é uma ignorância geral da profissão. A terceira incerteza seria sobre como diferenciar entre as duas primeiras. Engelhardt 13 apontou o caráter probabilístico do conhecimento científico. Sendo probabilístico, as certezas também o seriam, guardando, portanto, sempre uma margem de incerteza estrutural. Assim, frente à tamanha incerteza, observa-se um padrão de resposta por parte dos responsáveis pela assistência à saúde quando consideram que existem dilemas ou problemas morais relevantes envolvidos com seus objetos de trabalho: buscar aumentar os seus graus de certeza, ancorando-se em suas bases científicas.

A educação do futuro deveria enfrentar o problema do erro e da ilusão 14 . O maior erro se- ria subestimar o erro, e a maior ilusão seria subestimar a ilusão. O reconhecimento do erro e da ilusão, entretanto, é o mais difícil, já que eles não se reconhecem como tal. Entendendo a racionalidade como corretiva da percepção dos sentidos, deve-se, contudo, aceitar a idéia de que a racionalização é a perversão da racionalidade, uma vez que se crê perfeita por ser fundamentada em deduções e induções e é fechada, enquanto a racionalidade é aberta. Para Morin 14 , a racionalidade dialoga com o real e não é uma exclusividade da ciência ou do dito "Ocidente”. Daí que se deve reconhecer um princípio de incerteza racional, que é uma prevenção contra o erro e a ilusão.

A tecnificação do conceito de futilidade terapêutica e a tentativa de reduzir o processo de decisão sobre o tipo de cuidado que deve ser oferecido aos pacientes compõem um modo de evitar as difíceis questões morais relacionadas, que não desaparecem nem são respondidas com a sua tecnificação. Até porque as evidências que confeririam certeza a esse tipo de abordagem não são isentas de problemas de método, que, eventualmente, contaminam os resultados finais 15 . Além disso, há a impossibilidade de derivarmos valores de fatos, nos termos da Lei de Hume 6 .

Um exemplo da confusão entre argumentos da ordem científica em contraposição a argumentos morais é visto na discussão sobre futilidade/obstinação terapêutica e distanásia, marcada por uma certa devoção à tecnologia. Defende-se a introdução de "fundamentos racionais na alocação de recursos de cuidados de saúde considerando a limitada disponibilidade deles, o que determina necessariamente que algumas intervenções estão distantes de alguns indivíduos" 16 (p. 958). Um exemplo é a utilização de escores de avaliação do conjunto de sinais, sintomas e indicadores fisiológicos e, assim, pretensamente tornar-se possível elaborar um guia seguro quanto ao provável desenlace da situação clínica. Algo assim tornaria o conceito de futilidade terapêutica um conceito essencialmente técnico, resultado da avaliação preditiva tornada possível com os estudos sistemáticos semelhantes aos que são desenvolvidos na elaboração de diretrizes e protocolos clínicos 17,18.

Não se discute o sofrimento dos pacientes e das pessoas que lhes são afetivamente próximas, mas os recursos envolvidos nessa assistência, em uma opção implicitamente economicamente utilitarista. Assim, o atendimento ou não das necessidades dos pacientes no final da vida passa a ser simplesmente uma questão econômica - até quando vale a pena, financei- 
ramente, investir? Essa opção, entretanto, também é uma opção que deveria levar a uma reflexão ética - que, em geral, também não é feita.

No campo da Saúde Pública, o legítimo esforço para a maior racionalização e fundamentação das decisões com indicadores com razoável grau de validade e precisão permeia os debates no campo do planejamento há muitas décadas. Das experiências do planejamento normativo às modernas abordagens estratégicas, busca-se não só a racionalização desse processo de alocação de recursos no sentido de maior eficácia como também no de maior justiça. Tais esforços aproximaram os campos da Economia e o da Saúde Pública. Foram construídos alguns indicadores que teriam utilidade promovendo uma alocação mais racional dos recursos: o QALY (Quality-Adjusted Life Year) e o DALY (Disability-Adjusted Life Year). Os fundamentos e propósitos de ambos os indicadores são semelhantes: o QALY é a unidade de medida de utilidade que combina anos de vida ganhos como resultado de intervenções de saúde ou programas de assistência à saúde com um julgamento sobre a qualidade desses anos de vida, e o DALY "é um indicador que procura medir simultaneamente o impacto da mortalidade e dos problemas de saúde que afetam a qualidade de vida dos indivíduos (...) mede os anos de vida perdidos seja por morte prematura ou incapacidade em relação a uma esperança de vida ideal" 19 (p. 899).

A despeito das boas intenções que levaram à criação do QALY, do DALY e de outros tantos indicadores econômicos destinados a orientar racionalmente a alocação de recursos, eles estão longe de serem aceitos sem reservas pela comunidade científica. As críticas mais significativas vêm do campo da ética, embora não só 20, e derivam desde o reconhecimento de problemas éticos decorrentes dos métodos de pesquisa utilizados para construir o indicador - $\mathrm{o}$ “time trade off" teria problemas teóricos de validade e de consistência interna, o que tornaria inapropriado seu uso como uma medida exata 21 - como até mesmo o fato de pressupor que a vida de alguém com incapacidade tem menor valor/utilidade que a de alguém sem incapacidades, o que suscita, no mínimo, um largo debate ético 22 .

Nenhuma dessas questões é habitualmente discutida no âmbito dos cursos de graduação em saúde e só eventualmente surgem nos programas de pós-graduação. Os profissionais de saúde não estão preparados e nem atentos a essas questões. Não é a toa que a reação mais habitual seja a de negar a discussão, buscando saídas já predefinidas (como são os códigos de ética) ou tecnicistas. Por que não pensarmos no bem que se faria a essas pessoas que provavelmente serão afetadas negativamente em seus interesses quando incluídas em algum grupo que, na avaliação técnica, não "merece investimentos para sobreviver ou ter sua qualidade de vida melhorada" de tomar a decisão que achar mais adequada para sua vida, inclusive abreviá-la, se for o caso? Não se deve é permitir, por preconceito ou ignorância, que pessoas sejam condenadas a um fim de vida sofrível e não lhes sendo formalmente permitido optar sobre como ele será.

\section{Processo de morrer e formação do pessoal de saúde}

Não basta criar rotinas ou discutir formas eficazes de organizar os serviços se não atuamos na formação de quem estará nesses serviços. $\mathrm{O}$ processo de trabalho em saúde se realiza na relação entre aquele que necessita de assistência e o profissional. O trabalho não se expressa em um produto, ele é imediatamente consumido no momento de sua realização. O processo de formação, necessariamente, deve contribuir para o desenvolvimento de competências e habilidades específicas relacionadas com o cuidado em fim de vida. Ele deve considerar a compreensão da morte como evento da vida, parte integrante dela e não como algo que deva ser combatido não importa em que condições.

A medicina integra um conjunto de dispositivos que visam controlar a vida, disciplinar os corpos para o trabalho 23 e à acumulação de capital. Construindo um discurso sobre o normal e medicalizando o desviante, a medicina logrou estabelecer os padrões de funcionamento humano. Essa é a tônica das profissões de saúde. Nessa perspectiva, a morte tem sido o momento que lhe escapa algo que deve ser absolutamente afastado. Nesse sentido, introduzir a morte e o processo de morrer como temática para a formação dos profissionais de saúde pode parecer indevido, já que não atenderia à lógica imposta por esse entendimento do processo de trabalho em saúde. Daí que, mesmo entre profissionais bem intencionados que atuam no campo dos cuidados paliativos, já existe a discussão de se criar especialidades que foquem a assistência dos pacientes em fim de vida.

Esse questionamento mostra, mais uma vez, que é imperiosa a radicalização da transição paradigmática na área da saúde - do combate à doença e à morte ao cuidado. Instituir o cuidado como paradigma das profissões de saúde implica na emergência do sujeito, autônomo, 
senhor de sua história construída de forma compartilhada. Assim deve ser a história do fim da vida de cada um: dirigida pelo titular da vida ou a quem o poder de decidir for legitimamente atribuído, história construída de forma compartilhada com os profissionais de saúde que $\mathrm{o}$ assistem e com as pessoas mais próximas afetivamente. Nessa perspectiva, todas as opções devem estar legítima e legalmente disponíveis para os indivíduos, desde o cuidado intensivo até a eutanásia e o suicídio assistido.

A atitude de tomar a realidade nas mãos e de assumir o papel e a responsabilidade cabida na construção de uma história compartilhada significa conhecer, refletir sobre os melhores caminhos e agir no sentido de contribuir para um mundo mais saudável. Isso faz de cada pessoa envolvida no processo de trabalho em saúde um agente na construção de uma sociedade mais justa, na concretização do direito à saúde. Assumir essa responsabilidade implica em uma defesa sistemática da dignidade, não só da população que busca os serviços de saúde, mas também das pessoas envolvidas na prática profissional. Ocupar o papel e a responsabilidade de estar no mundo significa pensar na inclusão da humanidade como objeto de nossas preocupações morais, assumindo, para além do discurso retórico, o compromisso com o bem-estar dos indivíduos e das populações que podem ter (e freqüentemente têm) valores diferentes que os dos profissionais que o assistem, tornando o diálogo respeitoso como premissa

\section{Resumo}

A morte é uma questão implícita na prática e na formação dos profissionais da saúde. Há, todavia, uma tendência dos profissionais de saúde de restringir sua discussão aos aspectos meramente técnicos, seja na assistência à saúde, seja nas ações de saúde pública. Este artigo analisa a importância da adoção do cuidado de qualidade no fim de vida como norteador das práticas assistenciais e a necessidade de que o campo da Saúde Pública tome esses cuidados como objeto de reflexão e de pesquisas. Por fim, aponta-se para aspectos relevantes para a formação de profissionais de saúde para que sejam respeitadores das diferenças e comprometidos com o diálogo.

Cuidados Paliativos; Política de Saúde; Qualidade de Vida; Bioética para a realização de uma relação eticamente fundamentada.

É nessa perspectiva que se insere o cuidado no fim da vida: é algo que é pensado e realizado, em cada caso concreto, de forma compartilhada, entre seres autônomos que se respeitam e que constroem um processo de morrer no qual os profissionais, por meio de um conhecimento técnico, podem contribuir para que esse processo seja vivido dignamente com o mínimo de sofrimento para o próprio e seus cuidadores. No nível social, é uma opção radical na construção de um mundo mais justo e solidário.

É difícil, entretanto, afirmar que as reformas que vêm sendo implementadas no ensino das profissões de saúde já trilhem o caminho desejado de despertar a consciência e a sensibilidade moral e o compromisso com o respeito ao outro e com o diálogo, embora seja possível identificar, nos documentos oficiais que amparam os processos de reforma, a intenção de conferir à formação um forte componente humanístico 24 . Falta, entretanto, a compreensão que sem o enfrentamento das questões morais relacionadas com o processo de formação e a preocupação com a formação de pessoas autônomas e comprometidas com o respeito aos direitos humanos e com o diálogo não chegaremos a mudanças mais sustentáveis na qualidade da assistência e no respeito aos indivíduos que procuram assistência profissional no sistema de saúde brasileiro.

\section{Colaboradores}

Ambos os autores tiveram participação em todas as fases do artigo. 


\section{Referências}

1. Ariès P. História da morte no ocidente. Rio de Janeiro: Francisco Alves; 1977.

2. Floriani CA, Schramm FR. Atendimento domiciliar ao idoso: problema ou solução? Cad Saúde Pública 2004; 20:986-94.

3. Mori M. A bioética: sua natureza e história. Humanidades 1994; 9:332-41.

4. Schramm FR. A moralidade da biotecnociência: a bioética da proteção pode dar conta do impacto real e potencial das biotecnologias sobre a vida e/ou a qualidade de vida das pessoas humanas? In: Schramm FR, Rego S, Braz M, Palácios M, organizadores. Bioética, riscos e proteção. Rio de Janeiro: Editora UFRJ/Editora Fiocruz; 2005. p. 15-28.

5. Elias N. A sociedade dos indivíduos. Rio de Janeiro: Jorge Zahar Editor; 1994.

6. Siqueira-Batista R, Schramm FR. Eutanásia: pelas veredas da morte e da autonomia. Ciênc Saúde Coletiva 2004; 9:31-41.

7. Singer PA, Bowman KW. Quality end-of-life care: a global perspective. BMC Palliat Care 2002; 1. http://www.biomedcentral.com/1472-684X/1/4 (acessado em 08/Abr/2006).

8. Araújo CO. Brasil celebra o Dia Mundial de Cuidados Paliativos e Hospice e é um dos países mais atuantes nas comemorações. Prática Hospitalar 2005; 42. http://www.praticahospitalar.com.br/ pratica\%2042/pgs/materia\%2010-42.html (acessado em 18/Abr/2006).

9. Menezes RA. Em busca da boa morte. Rio de Janeiro: Editora Fiocruz/Garamond Universitária; 2004.

10. Ministério da Saúde. Política Nacional de Humanização. Humaniza SUS. 2004. http://portal. saude.gov.br/portal/arquivos/pdf/doc_base.pdf (acessado em 20/Abr/2006).

11. Mattos RA. Os sentidos da integralidade. In: Pinheiro R, Mattos RA, organizadores. Os sentidos da integralidade. Rio de Janeiro: Instituto de Medicina Social, Universidade do Estado do Rio de Janeiro; 2001. p. 39-64.

12. Fox RC. Training for uncertainty. In Merton RK, Reader GG, Kendall PL, editors. The student physician: introductory studies in the sociology of medical education. Cambridge: Harvard University Press; 1957. p. 207-41.
13. Engelhardt Jr. HT. Bioética do risco: enfrentando a finitude humana. In: Schramm FR, Rego S, Braz M, Palácios M, organizadores. Bioética, riscos e proteção. Rio de Janeiro: Editora UFRJ/Editora Fiocruz; 2005. p. 133-47.

14. Morin E. Os sete saberes necessários à educação do futuro. 3a Ed. São Paulo: Cortez/Brasília: Organização das Nações Unidas para a Educação, a Ciência e a Cultura; 2001.

15. Teres D, Lemeshow $\mathrm{S}$. Why severity models should be used with caution. Crit Care Clin 1994; 10:93110 .

16. Truog RD, Brock D, Cook D, Danis M, Luce JM, Rubenfeld GD, et al. Rationing in the intensive care unit. Crit Care Med 2006; 34:958-63.

17. Glance LG, Osler T, Shinozaki T. Intensive care unit prognostic scoring systems to predict death: a cost-effectiveness analysis. Crit Care Med 1998; 26:1842-9.

18. Bastos PG, Sun X, Wagner DP, Knaus WA, Zimmerman JE; The Brazil APACHE III Study Group. Application of the APACHE III prognostic system in Brazilian intensive care units: a prospective multicenter study. Intensive Care Med 1996; 22:56470.

19. Schramm JMA, Oliveira AF, Leite IC, Valente JG, Gadelha AMJ, Portela MC, et al. Transição epidemiológica e o estudo de carga de doença no Brasil. Ciênc Saúde Coletiva 2004; 9:897-908.

20. Almeida Filho N. O conceito de saúde: ponto cego da epidemiologia? Rev Bras Epidemiol 2000; 3:4-20.

21. Arnesen TM, Norheim OF. Quantifying quality of life for economic analysis: time out for time trade off. Med Humanit 2003; 29:81-6.

22. Lyttkens CH Time to disable DALYs? On the use of DALYs in health policy. http://www.nek.lu. se/ryde/luche99/Papers/lyttkens.pdf (acessado em 09/Abr/2006).

23. Foucault M. História da sexualidade. 8a Ed. Rio de Janeiro: Graal; 1985.

24. Rego S. A formação ética dos médicos. Rio de Janeiro: Editora Fiocruz; 2004.

Submetido em 24/Abr/2006

Aprovado em 24/Abr/2006 